\title{
Measurement Error and Statistics of Pairing Microphones for Directional Arrays in Hearing Aids
}

Warren, Daniel M. and King, Charles B.

Knowles Electronics

1151 Maplewood Drive, Itasca, Illinois 60143 (USA)

Hearing aids that have a microphone response that is dependent upon the direction from which the sound arrives have been shown to provide benefit in understanding speech in background noise for hearing impaired listeners in many situations [1]. The directional response acts like a spatial filter with an increased gain for sound coming from the forward direction (the usual direction the wearer is attending) over sounds coming from other directions. The small size of hearing aids is a challenge to making a directional system, requiring microphones that are closely matched in their response characteristics. Providing such closely matched pairs of microphones is a fascinating mathematics problem, involving probability, graph, statistical, and quality control theories.

\section{Directional Response}

The directional microphone response in hearing aids is most often implemented using two microphones separated on the body of the aid by some distance $d$. The directional response [3] is given by

$$
R(\theta)=g_{0} s_{0} e^{-j \frac{k d}{2} \cos \theta}+g_{1} s_{1} e^{j \frac{k d}{2} \cos \theta},
$$

where $s_{i}$ are the complex sensitivities of the two microphones, $g_{i}$ are the complex gains the hearing aid system applies to each microphone, $k=2 \pi f / c$ is the acoustic wavenumber at frequency $f$ for sound speed $c$, and $\theta$ is the angle from which the sound is arriving with respect to the line from microphone 0 to 1 . Modern hearing aids are quite small and narrow, so the spacing $d$ is typically about $1 \mathrm{~cm}$ on a horizontal line pointing forward when the aid is worn properly. With this close spacing, the system is working in the long wavelength limit $k d \ll 1$ across the useful frequency band of the hearing aid. Assuming that the sensitivities of the microphones are identical $\left(s_{0}=s_{1}\right)$ the gains $g_{i}$ can be chosen so the directional response follows the limiçon family of curves (Figure 1)

$$
R(\theta)=A+B \cos \theta,
$$

where $A$ and $B$ are real valued numbers. The limiçon curves have the highest value at $\theta=0$ (the forward direction) and have one or two nulls symmetrically between $\pm 90^{\circ}$ and $180^{\circ}$. Many hearing aids have adaptive systems that steer these nulls toward the loudest signal interfering with the sound coming from the front.
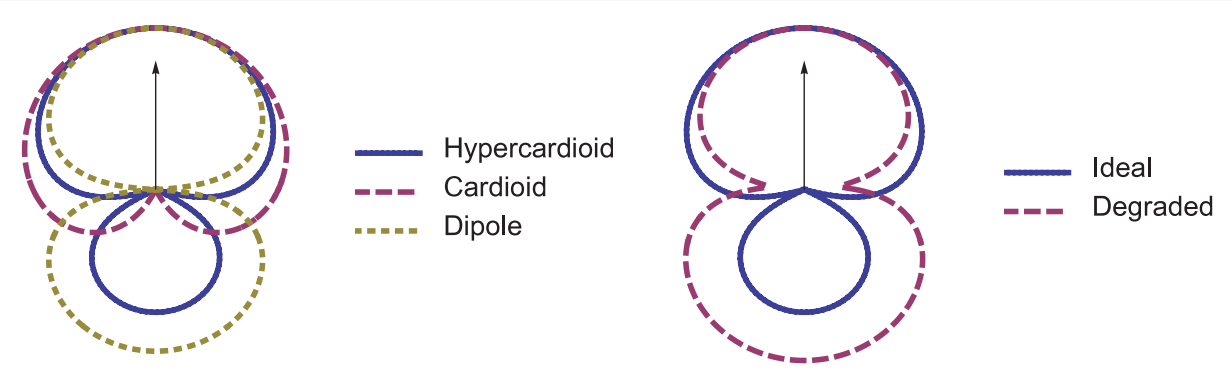

Figure 1: On the left are ideal directional responses showing different null angles. The figure on the right shows how a highly directional pattern can be degraded by a small mismatch between the microphones.

If the microphone responses are not identical, however, the directional response is

$$
R(\theta)=(A+B \cos \theta)+\frac{\delta}{2}(A+B \cos \theta)+j \frac{\delta B}{k d},
$$

where the sensitivity ratio $\delta=s_{1} / s_{0}$ is a complex-valued function of frequency. The first term is the expected response and the second term has the same shape so does not degrade the directivity. The third term, 
however, is an error term that degrades directivity. The error gets worse as $k d$ gets smaller, which occurs for small $d$ and lower frequencies. Figure 1 shows a degraded directional response at $200 \mathrm{~Hz}$, where a $-3.88 \mathrm{~Hz}$ difference in $f_{c}$ has caused $\delta=0.038 \mathrm{~dB},-1^{\circ}$.

\section{Microphone Response}

The sensitivity ratio $\delta$ is not an arbitrary function of frequency because microphone sensitivity is not an arbitrary function. A hearing aid microphone response can be well modeled as a filter with a first-order high-pass and a resonance

$$
s(f)=s_{m} \frac{j \frac{f}{f_{c}}}{1+j \frac{f}{f_{c}}}\left(1-\frac{f^{2}}{f_{0}^{2}}+j \frac{f}{Q f_{0}}\right)^{-1},
$$

where $s_{m}$ is the mid-band sensitivity, $f_{c}$ is the corner frequency of the high-pass, $f_{0}$ is the resonance frequency, and $Q$ is the quality factor of the resonance. When the sensitivity is plotted on a decibel scale, the numbers can be read right off the plot given that $M_{m}=20 \log _{10} s_{m}, Q=20 \log _{10} \Delta_{p}, \Delta_{p}$ is the difference in dB between the amplitude of the maximum and $M_{m}$, and $f_{c}$ is the frequency at which the low frequency sensitivity has dropped $3 \mathrm{~dB}$ below $M_{m}$. All parameters are real-valued. The facts that are relevant to this discussion are

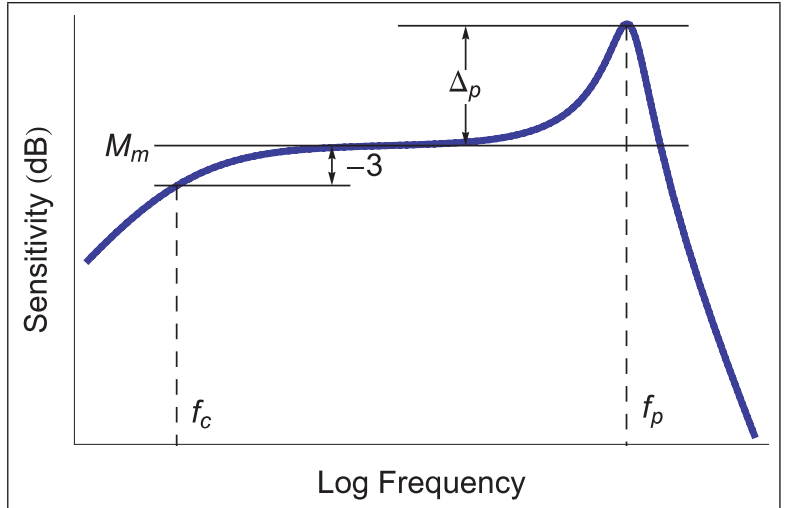

Figure 2: Plot of a typical microphone response diagramming the four degrees of freedom.
- A microphone has 4 degrees of freedom.

- The parameters describing the degrees of freedom are easily extracted from measured data.

- The parameters are independent and uncorrelated.

A couple of caveats: the corner frequency $f_{c}$ is not directly measurable. To determine $f_{c}$ would require sensitivity magnitude measurements taken at several low frequencies. It is time-consuming to collect lowfrequency data over sufficient periods to stabilize the measurement. It can be shown that a phase measurement $\phi$ at a single low frequency is strongly correlated to the corner frequency. At the high frequencies, the resonance frequency is not quite the frequency of the maximum value $\left(f_{0} \approx f_{p}\right.$, the frequency of $\left.\Delta_{p}\right)$, but it is mathematically related and strongly correlated.

\section{Matching Criteria}

\begin{tabular}{|ccccc|}
\hline & Quality & \multicolumn{3}{c|}{$\begin{array}{c}\text { Matching } \\
\text { Tolerance }\end{array}$} \\
& Limits & $\sigma$ & $|X-Y| \leq t$ & $t / \sigma$ \\
\hline$M_{m}(\mathrm{~dB})$ & $-58 \pm 3$ & 1.1 & 1.0 & 1.1 \\
$\phi\left({ }^{\circ}\right)$ & & 3.1 & 2.0 & 0.65 \\
$f_{p}(\mathrm{~Hz})$ & $4500-6000$ & 190 & 250 & 1.3 \\
$\Delta_{p}(\mathrm{~dB})$ & $9 \pm 3$ & 1.0 & 1.5 & 1.5 \\
\hline
\end{tabular}

Table 1: Specifications and distribution data for parameters used for matching a typical hearing aid microphone pair. The actual matching tolerance for the mid-band sensitivity $M_{m}$ is much tighter than shown here as discussed in the text. The phase $\phi$ is measured at $200 \mathrm{~Hz}$, and does not have quality specification limits.
Four real-values parameters $\left(M_{m}, \phi, f_{p}\right.$, and $\left.\Delta_{p}\right)$ completely characterize a microphone response, so matching these parameters between two microphones is necessary and sufficient to match the complex response throughout the frequency range. Fewer parameters would underdetermine the match, more would overdetermine the match. Overdetermining the match does not improve the quality of the match. Through analysis and simulation [5], it has been determined that to have high directivity across the usable frequency range of a typical hearing aid that the microphones should be matched according to the criteria in Table 1.

The directivity of the pair is very sensitive to differences in $M_{m}$ and require a matching tolerance far 
tighter than shown in Table 1. Fortunately it is easily corrected by calibrating the microphones in the hearing aid system at the time of manufacture. A modest gain or attenuation $g_{i}$ is applied to the appropriate microphone to equilibrate their mid-band sensitivity. Calibrating phase is a much more complicated process [4].

It can be seen in Table 1 that the matching tolerances are much tighter than the manufacturing quality specifications. Attempting to manufacture all microphones within the matching tolerances would drive the cost of the microphones beyond market acceptability. Instead, the matching parameters are measured (using our best measurement practices to minimize measurement error as discussed later) on all microphones manufactured in a batch, and pairs of microphones that comply to all the matching criteria are chosen and packaged as matched pairs.

\section{The Statistics of Matching}

The matching process has its own limitations. Intuitively, it is obvious that narrower matching tolerances means fewer matches within a lot. To quantify, we assume the measured parameters follow a normal distribution. This is not strictly true, but it is a good enough assumption to serve as a starting place to understanding the statistics of the matching problem.

In a normal distribution $X(\sigma)$ with standard deviation $\sigma$, the probability of value $x$ is given by the probability density function (PDF)

$$
p(X(\sigma) ; x)=\frac{1}{\sqrt{2 \pi} \sigma} e^{-\frac{x^{2}}{2 \sigma^{2}}},
$$

The probability of a value less than $x$ or between $x_{1} \leq x \leq x_{2}$ is given by two forms of the cumulative density function (CDF)

$$
c(X(\sigma) ; x)=\int_{-\infty}^{x} p(X(\sigma) ; y) d y=\frac{1}{2}\left(1+\operatorname{erf}\left(\frac{x}{\sqrt{2} \sigma}\right)\right) ; \quad c\left(X(\sigma) ; x_{1}, x_{2}\right)=\int_{x_{1}}^{x_{2}} p(X(\sigma) ; y) d y .
$$

where $\operatorname{erf}(x)$ is the error function. The probability density of the sum of two independent randomly distributed variables is the convolution integral of their density functions [2, $p$ 116],

$$
p(X+Y)=p(X) * p(Y)=\int_{-\infty}^{\infty} p(Y ; y) p(X ; w-y) d y .
$$

From the last two properties, it is easy to verify that for two normal distributions with the same standard deviations

$$
X(\sigma) \pm Y(\sigma)=W(\sqrt{2} \sigma)
$$

that is, the sum (or difference) of normally distributed values has a standard deviation of $\sqrt{2} \sigma$.

Now consider the matching problem, where we want to know the probability that two values match to within a tolerance $t$, or, what is the probability $P_{1}$ that $|X-Y| \leq t$ ? Writing that another way, $-t \leq X-Y \leq t$, it is obvious that the solution is given by the CDF

$$
P_{1}=c(X(\sigma)-Y(\sigma) ;-t, t)=c(W(\sqrt{2} \sigma) ;-t, t)=\operatorname{erf}\left(\frac{t}{2 \sigma}\right),
$$

When matching on several parameters, all matching criteria must simultaneously be true (an AND condition), so the probability of matching on all parameters is the product of the probability of individual parameters matching,

$$
P_{1}=\prod_{k=1}^{M} \operatorname{erf}\left(\frac{t_{k}}{2 \sigma_{k}}\right),
$$

where $M$ is the number of independent parameters determining a match. In a population of $N$ microphones, there are $N(N-1) / 2$ possible combinations of two microphones to check for conformity to all matching criteria, so the most likely number of matches found will be

$$
E=\frac{1}{2} N(N-1) P_{1}
$$

Sadly, this cannot predict the number of actual pairs of microphones we can pull from the lot because any one microphone can be a member of only one pair, as described below. However the matching probability $P_{1}$ can serve as a figure-of-merit for a proposed set of matching criteria. 


\section{Optimal Matching and Graph Theory}

Graph theory is a branch of discrete mathematics used to model pairwise relationships between items in a collection and is the perfect discipline for analyzing this problem. A graph is composed of vertices, often represented a dots, which may be connected by edges, represented by lines connecting the dots.

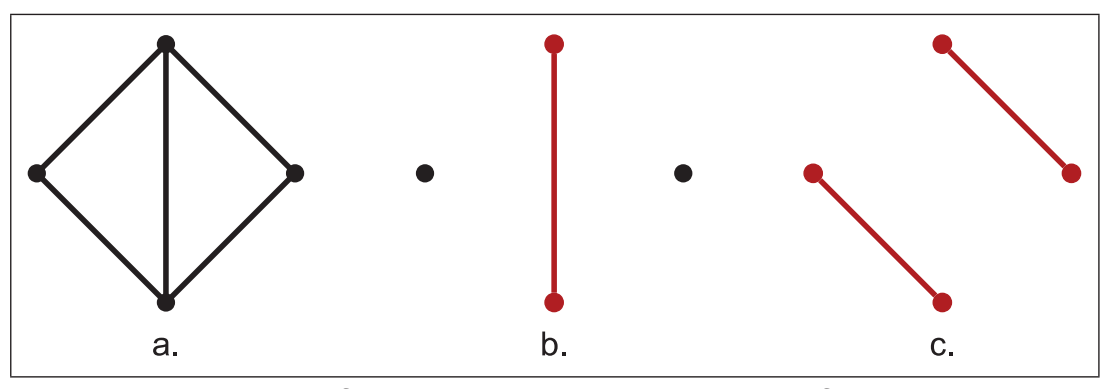

Figure 3: A graph of microphone matching among four microphones (a.) One strategy (b) results in a single pair being drawn, whereas a different strategy yields two pairs (c.) suspect that the number of pairs which can be removed depends strongly upon the choice of which matched microphones are paired. Figure 3 shows a small lot of microphones and two different strategies for choosing pairs, one which results in one more pair than the other.

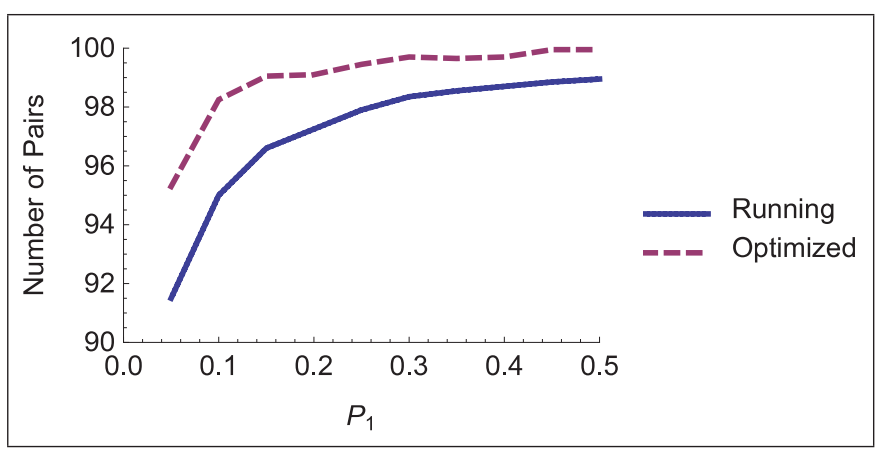

Figure 4: Results from simulations matching 200 microphones as a function of matching probability $P_{1}$. Two pairing strategies are shown, either selecting pairs as soon as a matching microphone is built ("Running") or the optimized pairing described in the text. become a pair. The third will be
are less obvious, but one begins to

For the matching problem, each microphone is represented by a vertex, and edges are drawn between vertices representing two matched microphones. The formula above predicts the number of edges drawn in the graph of $N$ vertices. It is immediately obvious that some microphone are not matched by any other in the lot and cannot be paired. There are also clusters of three matched microphones, only two of which can become a pair. The third will be
are less obvious, but one begins to Finding the strategy that maximizes the number of pairs pulled from a lot is a nonlinear integer optimization problem which in general are very difficult to solve. Fortunately, this specific problem can be approached by a relatively simple algorithm that results in an optimal or nearly optimal solution. The idea behind the solution comes from the game "Six Degrees of Kevin Bacon," in which the successful player recognizes early that some actors are particularly well connected, that is, they have appeared in many movies with other actors, including Mr. Bacon. The problem reduces to finding a shorter path to one of these well connected actors. If the rules of the game are altered so these actors cannot be used (taking John Lithgow or Elizabeth Shue out of play, for example) the game becomes much harder.

Conversely, to maximize the number of pairs found in the lot one should not remove well connected microphones. The strategy is to find the loneliest microphone and pair it with its loneliest neighbor. In terms of graph theory, the degree of a vertex is the number of edges connected to it, and the depth-one neighbors of a vertex are the vertices at the other end of those edges.

\section{- Find the vertex with the lowest non-zero degree}

- Find the depth-one neighbor of that vertex with the lowest non-zero degree

- Remove those two microphones from the lot and package as a pair

- Delete the two vertices and their edges from the graph

- Repeat until all vertices are degree zero 


\section{Measurement Error and Quality}

Our ability to match microphones based on measured parameters is limited by our knowledge of the true value of the parameters used to make the match. All measurements have some error, and as the tolerances get tight measurement error plays a larger role, and may result in rejection of good pairs or quality escapes if not carefully considered.

A test system is qualified using a standard Gauge Repeatability and Reproducibility (GR\&R) process, which compares the range of a measurement taken by a number of observers over a number of trials to the specified quality limits of the device being tested. The values in the R\&R study are assumed to represent samples from a normal distribution with standard deviation $\sigma_{m}$. The total range of the measured values is taken to be either the range which encompasses $99 \%$ of the distribution $\left(g_{c} \approx 5.15 \sigma_{m},\right)$ or $g_{c}=6 \sigma_{m}$, depending on the standard used. The GR\&R $g$ is the ratio of the measurement range to the product tolerance range $t_{p}$, often expressed as a percentage. Our factories report the GR\&R of all our test systems, from which we can extract the standard distribution of measurement error $\sigma_{m}=g t_{p} / g_{c}$. There are some arguments concerning the value of $t_{p}$ when it comes to matching, whether $t_{p}=t$ or $t_{p}=2 t$. Our convention is $t_{p}=t$. As long as same convention is followed by the person writing the GR\&R report and the person using this analysis, the following formulas will be correct.

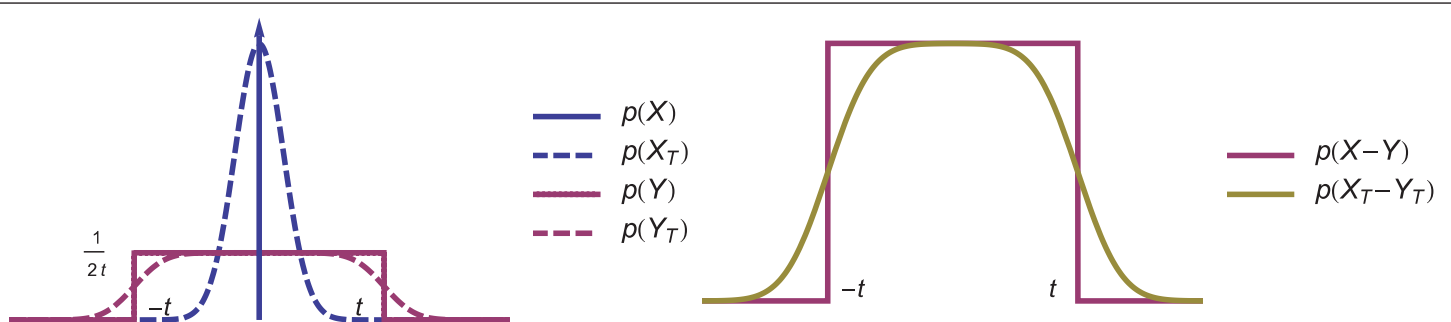

Figure 5: On the left, the distributions of measured and true values of $X$ and $Y$. On the right, the distribution of the measured and true difference between the values. The true values that fall outside the measured values are out of specification and considered quality escapes.

Consider the effect an unbiased, normally distributed measurement error $\epsilon$ will have on a population of paired microphones $(X, Y)$, which were paired based on measured values. The measured value of $X$ is arbitrary as it is only a reference for $Y$, so its probability density $p(X)=\delta(0)$, where $\delta(x)$ is the Dirac delta function of value 1 at $x$ and 0 elsewhere. Any microphone between $\pm t$ matches $X$, so $p(Y)$ is the PDF of a uniform distribution, or a boxcar function $2 t$ wide and $(2 t)^{-1}$ tall, as shown in Figure 5 . The true values $X_{T}$ and $Y_{T}$ can be found by convolving $X$ and $Y$ with error distribution $\epsilon$, also shown in Figure 5. Convolving $X_{T}$ with $Y_{T}$ yields the probability distribution of the true difference between the microphones. The convolutions can be performed by inspection by using the associative property of convolution,

$$
X_{T} * Y_{T}=\left(X * \epsilon\left(\sigma_{m}\right)\right) *\left(Y * \epsilon\left(\sigma_{m}\right)\right)=(X * Y) *\left(\epsilon\left(\sigma_{m}\right) * \epsilon\left(\sigma_{m}\right)\right)=(\delta(0) * Y) * \epsilon^{\prime}\left(\sqrt{2} \sigma_{m}\right)=Y * \epsilon^{\prime}\left(\sqrt{2} \sigma_{m}\right)
$$

where we have use the fact that the Dirac delta function $\delta$ is the identity for convolutions. The convolution integration is trivial using the CDF, thus

$$
p\left(X_{T}-Y_{T} ; x\right)=\frac{1}{4 t}\left(\operatorname{erf}\left(\frac{x+t}{2 \sigma_{m}}\right)-\operatorname{erf}\left(\frac{x-t}{2 \sigma_{m}}\right)\right)
$$

which is plotted in Figure 5.

The density function of the true value of the difference extends outside the match tolerance, implying that some fraction of pairs will not be matched within tolerance $\pm t$, which would be considered a quality escape. The quality level $Q$, which is the fraction of product that truly comply to the specified tolerance, is found by integrating the density function over the tolerance limits

$$
Q=\operatorname{erf}\left(\frac{g_{c}}{g}\right)+\frac{g}{g_{c} \sqrt{\pi}}\left(e^{-\left(g_{c} / g\right)^{2}}-1\right) \approx 1-\frac{g}{g_{c} \sqrt{\pi}},
$$

where we have expressed $\sigma_{m}$ in terms of $g$ and expanded the function as a series to the first term (after having noticed that the plot of the function is linear over the reasonable range of $g$ ). This is an extremely 
interesting expression, as it directly relates quality level to the GR\&R in a very simple way. Given that quality level and GR\&R are metrics commonly used in quality assurance, you would think you would see such expressions more often.

It is now elementary to determine the that a GR\&R of $9 \%$ relative to the matching tolerance is necessary in order to achieve a quality level of $99 \%$; that is, 1 in 100 pairs fall outside the matching tolerance. This is almost impossible to achieve in a testing system on a high volume production line for the tight matching tolerances discussed in this paper. However, it is possible to achieve an acceptable quality level in a test system with higher GR\&R by matching to tolerances tighter than specified (called guardbanding) and adding a second verification layer of quality checking that directly compares the output of two microphones exposed to the same sound input. The statistics of guardbanding and verification will be subjects of a different paper.

\section{Conclusions}

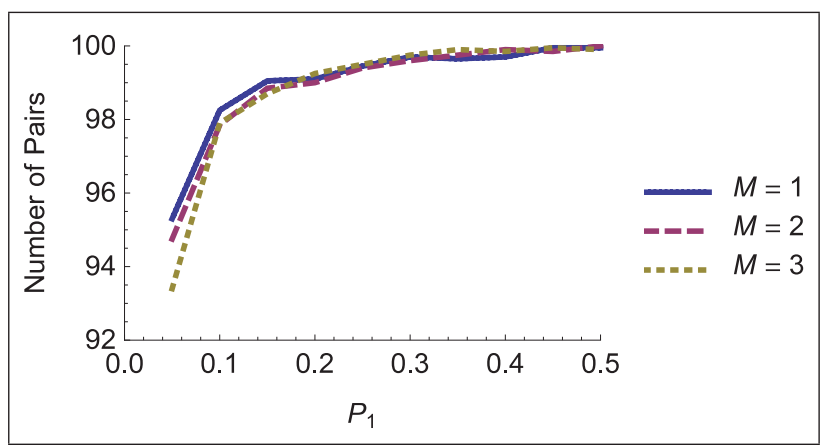

Figure 6: Results from simulations matching 200 microphones as a function of matching probability $P_{1}$ and varying number of matching parameters $M$, using the optimized pairing strategy.
Matching microphones based on a small number of measured parameters is a viable means of ensuring similar performance across a range of frequencies, thereby enabling high directionality despite close spacing of the microphones in the array. Reasonable yield is achievable as long as the matching probability $P_{1} \approx 0.1$ but begins to fall rapidly for smaller values (Figure 6.) One must also be aware of the limitations of the measurement system as matching criteria become tight. Means of determining the matching probability and relating metrics of the measurement system to the matching criteria have been provided.

It was believed early in the development of this theory that the matching probability $P_{1}$ could be used to predict matching yields for all matching situations or number of matching parameters $M$. From simulation results, it is apparent that this is reasonably true for $P_{1}>0.2$ (Figure 6.) However, as the matching probability becomes smaller, there is a dependence upon the number of matching parameters, where fewer pairs can be found in the same lot of microphones if all four parameters are used for matching. This is right where we are trying to work. For reference, the match probability for the practical microphones described in Table $1 P_{1}=0.09$. Further investigation will be made in this area.

\section{References}

[1] R. A. Bentler. Effectiveness of directional microphones and noise reduction schemes in hearing aids: A systematic review of the evidence. Journal of the American Academy of Audiology, 16:473-484(12), June 2005.

[2] P. Z. Peebles. Probability, Random Variables, and Random Signal Principles. Electrical and Computer Engineering, Communications and Signal Processing. McGraw-Hill, 3rd edition, 1980.

[3] S. C. Thompson. Directional patterns obtained from two or three microphones. Engineering Report 160, Knowles Electronics, September 2000.

[4] S. C. Thompson. Apparatus and method for matching the response of microphones in magnitude and phase. Patent 6654468, US Patent Office, November 2003.

[5] D. M. Warren and S. C. Thompson. Microphone matching for hybrid-order directional arrays in hearing aid applications. The Journal of the Acoustical Society of America, 113(4):2219-2219, 2003. 